\title{
Application Analysis of Palliative Nursing Intervention in Nursing Care of Elderly Cancer Patients
}

\author{
Shuhua $\mathrm{Li}^{1}$ \\ ${ }^{1}$ Weinan Vocational \& Technical College,Weinan,714000,China,Email: lishuhuachn@sina.com \\ lishuhuachn@sina.com
}

\begin{abstract}
With the trend of population aging, palliative care interventions are becoming more and more essential in the treatment of senile cancer diseases. This paper mainly researches palliative care intervention in elderly cancer patients by comparing the psychological、pain and death education of the three aspects of nursing philosophy and measures. It's very clear to find that the foreign palliative care interventions have strong pertinence, scientific and effective theoretical models and professional teams, and the death education and training system is relatively complete, which are the problems existing in China's palliative care and the development direction that should strengthen in the future.
\end{abstract}

Keywords: palliative care intervention, elderly cancer patients, psychology, pain, death education

\section{BACKGROUND}

Palliative care is a way to improve the quality of life of patients (young and old) and their families. In this method, the professional team adopts early identification, prevention, assessment, and treatment to alleviate the symptoms of patients; helps patients establish the correct concept of death and affirm the meaning of life; provides psychological and spiritual support systems for patients and their family, thereby improving the quality of life. Therefore, palliative care is comprehensive and dynamically developing the nursing model[1].

Nursing intervention is a series of nursing activities based on certain scientific theories and under the guidance of nursing diagnosis, according to predetermined intervention methods. Today, with the development of medicine, the concept of "prevention is greater than cure" is deeply rooted in the hearts of the people. In this environment, palliative care intervention has increasingly become the direction of international nursing research and development. Compared with developed countries, palliative care in China has problems such as late start, single method, immature system, and shortage of professionals. This paper will compare the differences in the application of domestic and foreign palliative care interventions in elderly patients through the three main aspects of "pain management", "death education" and "psychological care" in palliative care, and aim to find out the developmental problem of palliative care in China and provide certain ideas for future development.

\section{THE COMPARISONS BETWEEN DOMESTIC AND FOREIGN PALLIATIVE CARE IN PSYCHOLOGY CARE}

With the increase of age, the body function of the elderly declines, usually accompanied by cognitive decline, decreased appetite, hearing 、 memory and vision decline, and immune system symptoms[2]. These changes in physiology can make the sickness of elderly people concealed and the condition lasts for a long time. When the symptoms are obvious, it often means that the condition is in the middle and late-stage and is more serious, and the treatment effect will be unsatisfactory. Also, poor treatment effects, lack of understanding of the condition, fewer family members, lack of distractions in life, economic burden, lack of social function, and difficulty in adapting to changing roles may cause many psychological and mental problems such as anxiety, depression, loneliness, sensitivity, and excessive selfesteem [3]. These psychological problems will harm the treatment and recovery of the disease, thereby forming a vicious circle and accelerating the deterioration of the disease.

Palliative care psychological intervention is a new type of nursing intervention model, which aims to provide comprehensive and comprehensive psychological care for patients with malignant tumors in the middle and late stages. Through correct guidance, patients can face their illnesses calmly and spend the rest of their lives with dignity, thereby improving their quality of life[4]. Therefore, palliative care psychological intervention is an effective measure to solve the 
psychological problems of the elderly and improve the quality of care.

Among elderly patients, the most common psychological problems include depression, adjustment disorders, and anxiety disorders. At present, the main treatment methods of psychological intervention in palliative care abroad include cognitive behavioral therapy and belief intervention. The core of belief-based interventions is to focus on "here and now." In the setting of palliative care, patients live once a day and only consider the day' s events each time to better respond to their current situation. However, it is not effective for the psychological pressure of patients with advanced prostate cancer, cancer-specific pressure, and prostate-specific antigen anxiety. Belief interventions focus on four areas, namely attention, connection, gratitude, and goal. Specific measures include meditation and education about mindfulness, a guided visualization exercise, sympathetic listening courses, and reflection on core values[5].

The principle of psychological intervention in domestic palliative care is: it varies from the person, and gives care due to illness. First of all, nurses should establish a good relationship with patients,assess the patient's mental state according to the patient's personality, family background, cultural level,then choose appropriate psychological nursing interventions according to the patient's different psychological stages (depression, acceptance). Specific measures include: (1) Encourage patients to talk about their inner pressure and negative emotions, and provide support and comfort (application of palliative care interventions in patients with advanced cancer); (2) Guide patients to face the disease with a positive attitude and be optimistic Life; (3) Distract attention through some entertainment activities to relieve psychological pressure;(4) Use the method of psychological suggestion to euphemistically guide the patient to cooperate with treatment and receive various knowledge of treatment[6].

After long-term treatment, end-stage patients have relatively huger mental and economic pressures, which aggravate their emotional reactions such as fear, anxiety, depression, anger, guilt, and despair to a certain extent. And these negative emotions have adverse effects on the immune function of the body. Therefore, nurses should have a high degree of sympathy, understand the patient's mental activity rules, pay attention to their small wishes, and relieve the patient's negative emotions through communication and guidance, and enhance their confidence in fighting the disease[7].

\section{THE COMPARISONS BETWEEN DOMESTIC AND FOREIGN PALLIATIVE CARE IN PAIN MANAGEMENT}

Pain is a subjective feeling. Although diagnostic tests can help determine the cause of pain, there is no objective measurement or test that can fully quantify pain. Because of genetic differences, individual differences in pain perception, potential physiological differences, and superimposed emotional or spiritual effects, patients will have different feelings and descriptions of the same level of pain. Some patients still cannot pass on the history of pain. The patient's subjective description can provide clues to the origin of pain and help choose treatment options. But professionals should objectively assess the location, duration, nature, severity, aggravating and mitigating factors, treatment methods, functional activities, emotional and sleep disorders of the pain quantitative assessment[8].

Pain is the most common symptom in cancer patients, and it is also the most unbearable. $60 \%$ to $90 \%$ of patients with advanced cancer complained of severe pain, and about $25 \%$ of them had no effective relief of severe pain until death. Therefore, giving patients analgesics regularly will contribute to control the pain effectively before or at the beginning, help avoid the gradual increase in the dose, and will reduce the patient's psychological fear of pain, and affect the effect of psychological care. And it can allow patients to spend the last time of life without pain[9].

The WHO has developed a three-step "ladder" for reducing the pain of adult cancer, as shown in figure 1 . If pain occurs, the medication should be taken orally in time, and the order of medication is as follows: (1). Nonopioids (aspirin and paracetamol); (2). If necessary, take mild opioids (codeine); (3). Take strong opioids Drugs, such as morphine, until the patient no longer has pain. In addition, in order to relieve the patients' fear and anxiety, additional drugs-"adjuvants" should be used.

To help patients avoid pain, they should be administered "on time", that is, every 3-6 hours, rather than "on-demand". The three-step method of administering the right dosage at the right time is cheap and effective, and the pain relief rate can be as high as $80-90 \%$. If the drug is not fully effective, surgical intervention with appropriate nerves can further reduce pain. For childhood cancer pain, WHO recommends a two-level ladder[10].

\section{WHO's Pain Relief Ladder}

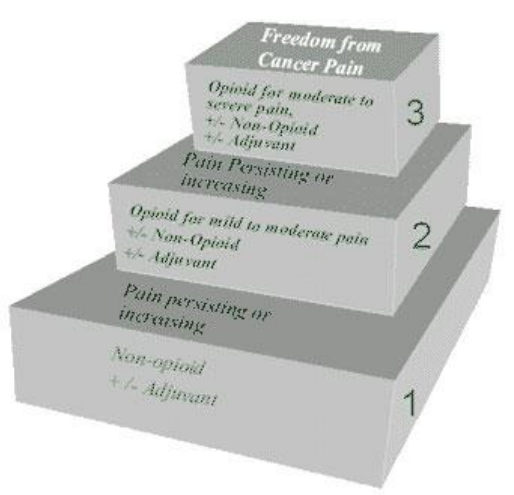

Figure 1.WHO’s Pain Relief Ladder 


\subsection{Pain care models abroad}

Palliative care interventions for pain in foreign countries are generally divided into three steps: (1) A professional team composed of nurses, doctors, and pharmacists conducts a preliminary assessment of patients' pain symptoms and a second objective assessment of the scale through conversations. Commonly used pain assessment scales are ICU Nonverbal Pain Scale, Pain Behavior Scale, and Pain Observation Tool for Critically Ill Patients. Ten nonverbal pain scales include the Facial Pain Scale and Umbek Face Pain Rating Scale[11].(2) Pain management is carried out according to the actual situation of the patient. Pain management divides into drug management and non-drug management. Drug management bases on the World Health Organization's pain ladder for medication treatment[12].Non-drug management mainly includes pain relief according to the pain gate theory, including psychotherapy, music therapy, massage and acupuncture pain relief, etc; exert family and social support to control the pain of cancer patients. (3) Relieve psychological and spiritual problems caused by pain. Pain intervention of palliative care runs through the treatment of the entire disease, and even when the patient has no pain symptoms, strictly abide by the five principles: (1) the principle of appropriate amount (2) the principle of application (3) the first principle of oral administration (4) regular medication (5) graded management[13].

\subsection{Pain care models in domestic}

By contrast, domestic pain care mainly refers to the steps of the stepwise analgesia formulated by the WHO and adopts multiple modes of administration such as subcutaneous injection, oral administration, and rectal administration to give patients regular and quantitative analgesic treatment. In the case of the ineffectiveness of analgesic treatment, the pain can be relieved by blocking and destroying the nerve pain pathway[14]. Radiotherapy is mainly using for bone metastasis of advanced tumors, local tumor compression, etc. and other symptomatic treatments to alleviate the patient' $\mathrm{s}$ fear and prompt them to treat diseases and correctly[15].

\section{THE COMPARISONS BETWEEN DOMESTIC AND FOREIGN PALLIATIVE CARE IN DEATH EDUCATION}

Death attitude refers to the evaluative and stable internal psychological tendency held by an individual when responding to death stimuli. According to previous studies, death attitudes can generally include death fear, death anxiety, death avoidance, and death acceptance include neutral death acceptance, approach-oriented death acceptance, and escape-oriented death acceptance[16]. The elderly have a high morbidity rate and a relatively high mortality rate. In China, only a few elderly people think that death is a normal thing and can be accepted frankly, while most elderly people still feel fear and anxiety in the face of death. The psychological problems that bring, such as fear of becoming a burden, loss of self-control, and problems related to personal relationships and meaning in life, will cause many elderly people to adopt a negative attitude of avoidance or even suicide[17]. The negative emotions and attitudes of the hospitalized elderly towards death were significantly higher than those of healthy people and patients with chronic diseases. The palliative care death education intervention measures help make the elderly understand that death is a normal and unavoidable thing, so that they can actively accept death, cherish life, respect life, actively cooperate with treatment, and improve the quality of life[18].

Related measures abroad are mainly meaning-based interventions, including looking back to the past, establishing the correct concept of life and death, seeking the meaning of life, and realizing personal value. Professionally trained nurses or palliative teams will use face-to-face communication with patients to review the bravest, most meaningful, and happiest events in their lives to help them affirm the value of their lives[19]; then discuss the handling of death in public Process and ethical issues, including euthanasia, corpse disposal, suicide, and disputes in living wills, etc., to help patients cope with loss and grief, reset life goals, reformulate a healthy and effective lifestyle, calmly face lost opportunities, and live actively in uncertainty[20].

The death education measures in domestic palliative care interventions mainly include four aspects: (1) To understand patients' views on death, outlook on life, world outlook, and values through videos, books, group discussions, etc[21] (2) Choose targeted Sex, historical figures or heroes similar to the patient enlighten the patient to face death correctly (3) Recalling happy, beautiful or fulfilling things with the patient and family members in the past will satisfy the patient' $s$ selfesteem and honor[22].(4) Teach patients to understand the connotation and meaning of death through videos, pictures, and brochures, so as to ensure that patients can open their hearts and correctly recognize and accept death[23].

\section{CONCLUSION}

By comparing the literature, it is found that the main differences of palliative care intervention between China and foreign countries regarding psychological intervention, pain management, and death education are:

(1) The psychological intervention measures in foreign countries aim to specific symptoms at different levels. These measures involve a wide range and strong pertinence and can be measured through randomized controlled experiments; while domestic measures are relatively general, and the family background 、 education level cannot be scientifically tested due to lack of scales. Evaluation classification, while the comfort and 
support methods do not describe the support of scientific theoretical models and specific measures. The palliative care interventions applied to patients with different diseases in the middle and late stages are similar and lack specificity. Therefore, it is particularly important and urgent to find supporting theoretical models of psychological interventions supporting palliative care and to refine specific measures.

(2) Domestic pain management lacks a professional team for pain assessment, the management method is single, and palliative care interventions are rarely conducted for the psychological and spiritual problems caused by pain.

(3) Although there are many death education methods for patients and their families in my country, and the content is relatively rich, it ignores the systematic training of death education for nurses themselves, including the emotional processing and psychological adjustment of nurses in death education. Effective communication methods and the cultivation of empathy ability, etc., and the lack of this aspect will reduce the quality of clinical death education and affect the patient ' s quality of life in the end-stage.

In the future, we can promote the development of palliative care if we focus on and develop palliative care team building, pain assessment scales, death education training models, and specific psychological interventions. As a result, the quality of life and happiness of elderly patients will be significantly improved in our country.

\section{REFERENCES}

[1] https://www.who.int/zh/news-room/factsheets/detail/palliative-care

[2] Xia Li,Zhu Kun,Wang Mingxuan,Peng Yan.Common psychological problems of elderly patients and nursing countermeasures[J].Modern Health Care,2015(10):262.

[3] Jing Miao.Psychological characteristics of elderly patients and nursing countermeasures[J]. Changjiang Series,2020(25):98+100.

[4] LI Yuling;GUO Liangyu;GUAN Yuhua;LIU Sa. Effect of palliative psychological intervention on psychological state, selfefficiency and quality of life of patients with orthopedic malignant tumors[J].ONCOLOGY PROGRESS, Sep2019, Vol.17, No.17

[5] von Blanckenburg P, Leppin N. Psychological interventions in palliative care $[\mathrm{J}]$. Curr Opin Psychiatry. 2018 Sep;31(5):389-395.

[6] Zhang Lun Zhang Hong Wu Lanhua Application effect of palliative nursing intervention in patients with advanced cancer[J] Contemporary Nurses: Academic Edition (Mid-term)" 2017, Issue 7, 85-87, 3 pages in total

[7] Cui Hong, Meng Xianlu, Ru Yanfen Palliative treatment and nursing intervention for patients with malignant tumors"Guide to Chinese Medicine" 2011 Vol. 18 163-164, 2 pages
[8] Bandieri E, Sichetti D, Romero M, et al. Impact of early access to a palliative/supportive care intervention on pain management in patients with cancer[J]. Annals of Oncology Official Journal of the European Society for Medical Oncology, 2012, 23(8):2016.

[9] Li Zihui, Gong Yin. Application of palliative nursing intervention in patients with advanced cancer $[\mathrm{J}]$. Contemporary Nurses (Academic Edition), 2010.

[10] https://www.who.int/cancer/palliative/painladder/e $\mathrm{n} /$

[11] Impact of early access to a palliative /supportive care intervention on pain management in patients with cancer

[12] Boesch J M. Advances in Pain Management: Palliative Care Applications[J]. Veterinary Clinics of North America Small Animal Practice, 2019, 49(3):445-461.

[13] Sheri, M, Kittelson, et al. Palliative Care Symptom Management[J]. Critical Care Nursing Clinics of North America, 2015.

[14] Luo Hongmin, Ge Hongxia. The basic content and model of palliative care[J]. Shandong Medicine, 2009, 49(045):114-114.

[15] $\mathrm{Hu}$ Zhi. The effect of palliative care intervention in patients with advanced cancer[J]. Diet Health Care, 2018, 005(018):198.

[16] Guo Hui, Ma Yan, Cheng Yang, et al. Research progress of elderly death attitudes and their assessment tools[J]. Nursing Research, 2020, v.34; No.651(07):107-111.

[17] Mao Jiayu Ren Kai Xu Xiaomin Chen Yue Fan Yujuan.Research Progress on Influencing Factors of Death Attitude of the Elderly in China[J].The Guide of Science \& Education2020, (18)187-188

[18] Guan Xiaoqing, Wang Xichao, Yu Xiuhua, Guan Yanzhu.Promote death with dignity from death education to improve the quality of the elderly at the end of life [J].Chinese Journal of Gerontology2019, 39(23)

[19] (Lima R, Bergold LB, Souza JDF, Barbosa GS, Ferreira MA. Death education: sensibility for caregiving. Rev Bras Enferm. 2018;71(suppl 4):1779-1784.

[20] Zhao SX, Qiang WM, Zheng XN, Luo ZQ. Development of death education training content for adult cancer patients: A mixed methods study. [J] Clin Nurs. 2018 Dec;27(23-24)

[21] Li Zihui, Gong Yin. Application of palliative nursing intervention in patients with advanced cancer[J]. Contemporary Nurses(Academic Edition), 2010(07):43-44. 【 22 】 Zhao Ying. Application of high-quality nursing concept in patients with advanced cancer[J]. Digest of World Latest Medical Information, 2016.

[22] Xu Rong. Analysis of the effect of palliative nursing intervention of death education on the poor psychology and quality of life of patients with advanced gastric cancer[J]. Electronic Journal of Practical Clinical Nursing, 2019, 4(33). 